depth of 590 feet. This is three times the depth of the Arctic minimum. The difference is due to the vast mass of the Antarctic icebergs in comparison with the hummocks.

Below this cold stratum to a depth of about 6,500 feet, extends a stratum of water warmer than $32 \mathrm{deg}$. F., having a uniform salinity of 3.46 per cent. The same perature gradually diminishes to $31.3 \mathrm{deg}$. F.

perature gradually diminishes to $31.3 \mathrm{deg}$. $F$.

We find here, then, a warm stratum between two cold strata, as in the Arctic. The upper cold stratum is
evidently moving northward, while the warmer but denser intermediate stratum consists of water flowing southward from warmer regions. The force deduced from the distribution of salinity and density at the surface is directed northward, but the prevailing winds, aided by the effect of the earth's rotation, deflect the surface current toward the west. This current is finally merged in the eastward current which marks the limit of the Antarctic circulation, at about $50 \mathrm{deg}$. S. lat The density of the surface water diminishes in goin Ther northward or southward from this line

The Antarctic ice is partly marine and partly terres- chiefly of blue ice formed along the shore. Antarctic icebergs are seldom encountered north of latitude 4.5, but
in 1894, the remnants of one were seen in $261 / 2 \mathrm{deg}$. S. lat. Our knowledge of the polar seas will almost certainly
Our sents of Our knowledge of the polar seas will almost certainly
be improved in a few years. A ship drifting with the be improved in a few years. A ship drifting with the
ice is in the best possible condition for observing the ice is in the best possible condition for observing the
physics of the sea. The expedition headed by Roald Amundsen will start from Bering Strait in the autumn of 1911, equipped with the most improved oceanographic apparatus. The plan is to cross the Arctic basin as near the pole as possible. Antarctic expeditions are also projected.

\title{
Characteristic Features of Rocky Deserts
}

\author{
Relation of Protective Crust to Ravine Formation
}

\section{By Dr. H. Burmester}

IN every rocky desert are found two distinctive features which form characteristic phenomena of the wide, barren landscape beaten upon by the fierce sun of the
sub-tropics: the so-called tropic protective crust and the intricately winding dry valleys with smooth, steep sides known as wadis.

These phenomena have hitherto been regarded as separate and dissociated in cause, but recent investigations have clearly demonstrated their close connection and interdependence.

The term "tropic protective crust" is used to designate the thin hard coating found upon all desert rocks. This coating, due to the intense dry heat, consists of a laye from the rock itself by scratching with a sharp instrufrom

According to the investigations of Mr. J. Walther, a yellow color shows a newly formed crust containing a hydrated iron oxide, a red color shows an older crust
which has been changed into iron oxide by loss of water, which has been changed into iron oxide by loss of water, The a gray tint shows the presence of manganese oxide.
The colors of the crust vary between deep black, clear gray, and all shades of brown, which generally shows gray, and all shades of brown, which generally shows the presence of silicic acid, the
richer being the crust in this acid.

The second marked phenomenon of these deserts are the ravines cut deep in the horizontal plateaus, inclosed by lofty, unscalable walls, and often ending in a steep
blind wall after long extents, measured by journeys of hours or even days

The desert east of the Nile valley, beginning near Cairo, is a characteristic rocky desert and affords an admirable as well as accessible field of observation.

1. INVESTIGATION OF EFFECT OF PROTECTIVE CRUST ON

In the desert a distinct difference is to be noted between stone exposed to direct sun and that which re ceives little or no sunshine.

The first is covered with a crust which is harder than the mother stone, and is severely eroded on the surface, to which fact is due the name tropic protective crust.

This crust or rind is formed wherever direct sun rays
This fact is due the name tropic protective crust. fall, but also creeps into tiny superficial cracks into which fall, but also creeps into tiny superficial cracks into which
no rays penetrate. It would thus seem to be due to the no rays penetrate. It would thus seem to be due to the
heating of the stone, which favors a chemical combinaheating of the stone, which favors a chemical combina-
tion of the stone with the iron and manganese salts of the atmosphere.

Those portions of the stone lying in shadow, on the other hand, are not protected by a crust against superother hand, are not protected by a crust against super-
ficial erosion. Hence the moisture of the night dew or an occasional rain remains longer and chemical erosion proceeds unhindered.

Erosion therefore begins in the shadowed part forming tiny cracks or projections which gradually increase inward until a cavity is formed in the side or top, or the projecting $\mathrm{p}$

own weight.
This is called erosion from within outwards. When a arge piece of rock forming such a projection breaks off a protective crust is at once formed on the exposed surface of the breach. The fallen block lying on the ground shows the protective crust on every side, but betrays inner cavities which have eroded outward so as to break through the crust, in which case the crust extends in patches from the roof of the cavity.

The pendent parts shadow the bottom of the cavities formed by them and therefore accelerate the erosion. But these fragments and projections owe their existence to the protective crust since this preserves them from too rapid decay.

Often, indeed, the mother stone is entirely eroded, and only the harder crust still hangs to the rock as the roof or sidewall of a cavity.

or sidewall of a cavity.
Thus we that the term "protective" crust is accurate only in that the stone immediately underlying it is proonly in that the stone immediately underlying it is pro-
tected. On the contrary, the crust favors the shade tected. On the contrary, the crust favors the shade
erosion which is the dominating factor in the desert, since erosion which is the dominating factor in the desert, since
through its greater hardness and durability it allows through its greater hardness and durability it allows
such shadow-making portions of the rock as the roofs such shadow-making portions of the rock as the roofs
or walls of caves to stand longer than they otherwise would.

Since the crust favors the dominant cause concerned in the characteristic rock forms of the desert, by logical
deduction it must be a significant factor in the formation of the wadis.

It is not, however, here implied that the crust is a necessary feature of erosion, since crusted and noneroded rocks are found.
In order to discover whether there was a "weathe side" in the desert, I examined Egyptian architecture. In the desert itself there are too many factors con-
cerned for a definite result to be reached. The direction cerned for a definite result to be reached. The direction of the wind and the hardness of the stone are determining factors, and moreover it is difficult to find a block of an undoubtedly synct

But it is a well-known fact that the stones employed in Egyptian buildings show crusts after 6,000 years.

One of the best known examples of this is the dam discovered by Schweinfurth in the Wadi Geraui at Heluan The blocks used there, which have an eastern exposure, are only slightly attacked on top and show a marke browness; yet a distinct

For our purpose how

For our purpose, however, the pyramids offer the best example, being exposed to every point of the compass. Of the pyramids at Ghiseh only the Chefren still shows portion of the original covering near the summit. The
others show only eroded blocks, the time of whose exothers show only eroded
posure is undiscoverable.

All four sides of the Chepen pyramid have an equally brown tint, showing an equal amount of erosion. The vertical summer sun, therefore, has strength enough to develop the crust even on the north side.

This facilitates the study of the wadis, since in in vestigating the crust erosion the orientation of the rocks
may be disregarded without its becoming a serious source may be dis

of error.
If we look at the Sphinx we observe that on the back of the head there is a strongly marked crust and obvious erosion has begun, while on the eastward-looking face there is little crust and little erosion. It seems to re-
main true that where there is little crust there is littl erosion.

$\Lambda$ special form of the crust is here referred to, in which on normal brown-crusted rock a slag-like applied crust appears in spots. This is black and always raised in relief. If such a stone is cut in sections there is usually found lying under these accretions darker stone containing lumps and hollows, or else arranged in layers. The hardness tests show it to consist of hornstone injections in chalk, which explains the dark color since this gains in darkness as it gains in silicic acid. It is not unusual for hornstone to be found in localities where fossilized wood is often found. In any case we se that harder stone raised in relief lies opposite crustcovered chalk.

When the chalk weathers its protective crust does not completely resist the outer erosion, but renews itself constantly much like the human skin without showing definite stantly

In this process of renewal it takes material from the mother stone and oc

This This erosion, which is naturally very slow, proceeds
side by side with the much more rapid and important hadow erosion.

The crust does not always work continuously into the rock. There are cases in which the crust breaks loose from the rock in layers-the process of desquamation. In
such instances the action is ascribed to solutions of salt which have filtered in and then crystallized. WADIS.

The extensive course of all wadis suggests water as the creative agent. The question is whether the quantity of water now available is sufficient to have caused th
or whether a greater precipitation formerly existed.

For the Sinai desert a pluvial period seems indicated. Hume concludes from marked boulder and pebble deposits that great snow fields existed in former ages. He considers it certain for Sinai that now in the pebble deposits of the wadi mouths the denudation exceeds the deposit, and that therefore smaller streams now dig their beds in the pebble deposits washed down by former mighty floods.

Direct conclusions as to the Nile valley cannot be drawn from this, but close observation of wadi formation gives various indications that water erosion has had a share in modeling the relief of the desert, and that to reater extent formerly than now.

Take the Wadi Hof, for example. It is at once obvious that we find ourselves in a valley where water has
been active. The ground consists of rounded grains of been active. The ground consists of rounded grains of
sand. If there are contiguous walls we find definite sand. If there are contiguous walls we find definite
water erosion grooves, with clearly defined wave marks. Here we find white eroded channels. Yonder we find heaped-up piles of rubbish.

Now let us direct our gaze to a bank of hard chalk over which erosive masses of water have undoubtedly revivifyinen some rare rainfall has sent down its the water must. On this bank we find at spots where crust, while wherever the water has dry furrows with violent deluges not the slightest remnant of the brown Thus we see that in many places the water has gained the victory, in others the crust has won the day.

In order to study better this conflict between crust and water I investigated the chalk banks in the branch wadis leading off from the main wadi, since there in all probability the crust action would be greater because of the smaller quantities of water.

This conjecture was verified, for without exception the round of the branch wadis showed, wherever hard chalk banks were present, dark crust above earlier erosion marks. In the foregoing $I$ have sought to present a series of erosion phenomena which give an impression that the
condition of the desert valleys of the localities considered cannot have been caused by such quantities of water as exist at present. The conflict between the water and the crust gave us
the clue, and showed how in the branch wadis the crust formation had the upper hand, while only in the main ravines could sufficient amounts of
fectually oppose the crust formation.

This struggle was active when mighty floods still oshed through the valleys; but slowly the waters diminshed and the crust won the upper hand.

The eroded chalk banks show positively that to-day the crust action is greater in extent than water erosion, part of thed worn by the rain water occupies only a small parts of the broken stone coated with the brown crust are not partially washed away again as we saw them in the main wadis.

If we observe upon a flat surface the formation of a tation which lead to small depressions and mark the course of the wadi. It is in these channels that the the runs when it rains, forming pools and occasioning the growth of vegetation.

The roots of these plants preserve the moisture longer,
The The roots of these plants preserve the moisture longer,
and therefore give rise to more rapid erosion in their and therefore give rise to more rapid erosion in their
neighborhood than in dry localities, and thus assist the neighborhood than in dry localities, and thus assist the
destructive action of chemical agencies later. At some point a projection is formed, cavities begin to be enlarged, and we have the commencement of a wadi.

The essential thing is, in my opinion, the co-operative action of vegetation and water, which loosens or disintegrates long strips of the desert and makes them more susceptible to subterranean erosion later.

That water is present in the sub-soil of the wadis is proved by the cisterns which are always found in the
course of the valleys. But their excavation is continued at the present day essentially by crust erosion and the formation of projections.

But the apparent conclusion that no valleys of long extent can now be formed without kettle-shaped cavities is not justified. Let us examine more closely the erosion in the wadis. We know that huge blocks fall through being hollowed out and help to widen the wadi, their fragments forming the rubbish of the sloping sides. On the one hand cavities will be formed, on the other the valley will be cut back. But since in the backward lengthening weathering by the influence of vepared for subsequent weathering by the influence of vegetation and subter-
will be considerably more rapid.-Translated for the Scientific American Supplement from Globus.

Excavations in the Ruins of Abasside Palace Archeological work of some interest was carried out by an expedition sent to Turkey from France and headed by M. Henry Viollet. He made excavations with the per-
mission of the Turkish government in the ruins of the mission of the Turkish government in the ruins of the first half of the ninth century of our era. It is a unique specimen of the architecture of this period which is still very obscure. The present palace was the residence of
the Arab caliphs who had been expelled from Bagdad the Arab caliphs who had been expelled from Bagdad
for a certain period on acount of military seditions. The
archæologist has now returned to Paris and made a report to the Academy on the subject. 